\title{
MATHEMATIC SIMULATION FOR UPPER DENTAL ARCH IN PRIMARY TEETH OCCLUSION
}

\section{Michael Porfiriadis, Sergei Dmitrienko, Dmitry Domenyuk, Gasan Budaychiev}

The need for studying individual features of the maxillofacial structure at orthodontic and prosthetic treatment has been proven by many authors, both national and foreign ones $[1,4]$. There has been a correlation identified between the shape, the dimensions of the dental arches, and the craniofacial morphometric parameters through different ages $[2,3]$. The HawleyHerber-Herbst method employed for graphic imaging of the dental arch has proven to contain certain faults, due to which correction factors have been developed taking into account the difference between the segment arch length and the length of the chord limiting the segment arch $[5,6]$. As far as the primary occlusion is concerned, the Schwarz method is recommended, based on the assumption that the dental arches shape through this stage of development is more like a semicir-cle, whose diameter is the dental arch width between the vestibular surfaces of the second primary molars. The methods for constructing dental arches proposed by other authors rely on the segments measurement; however, establishing correlation links between the lateral and anterior segments of the dental arches is not always available.

Arm. Design and explanation regarding the mathematic simulation method for the upper dental arch shape in children in the primary occlusion.

Materials and methods. The biometric study was performed on jaw die-stone models of 83 children who were on the completed primary bite stage (aged 4-6), and whose physiological occlusion corresponded the said age. On the stone models, the dental arches transversal dimensions were taken between the primary canines and second primary molars. The conventional lines connecting the points on the canines and the molars were used as a guide for measuring the depth of the anterior dental arch and the total depth of the arch, respectively. The frontal canine diagonal was taken from the central point to the point on the canine, while the frontal-distal distance reached the point level on the second primary molar. Odontometry was employed to identi-fy the anterior segment length and the dental arch length, as the sum of the width of the tooth crowns constituting the corresponding arches.
RESUlTS AND Discussion. The linear measurements of the dental arches have shown that the width of the dental arch between the canines was $34.01 \pm 0.56 \mathrm{~mm}$, while the depth of the anterior segment was $9.46 \pm 0.51 \mathrm{~mm}$. This data allowed determining the circle diameter for the placement of the six anterior primary occlusion teeth. Building a dental arch takes data about the width and depth, which, on the average, were in the said group equal to 49.79 $\pm 0.41 \mathrm{~mm}$ and $25.56 \pm 0.31 \mathrm{~mm}$, respectively. The diagonal from the interincisal point to the canine was within the range of $18.34 \pm 0.32 \mathrm{~mm}$, whereas the dental arches diagonal dimensions were $35.52 \pm 0.45 \mathrm{~mm}$. The data is average and can be used for graphical imaging of the average dental arches, which does not meet the contemporary requirements of orthodontics and patient-centered healthcare. Due to this, a method for constructing a dental arch diagram based on the child's in-dividual features was developed. The ratio of the arch width between the canines to the central angle sine allowed determining the circle diameter for the front teeth. The central angle, accord-ing to the laws of the circle geometry, was the ratio of the arch depth double arctangent to the width between the primary canines. From the top point of the circle, which corresponded to the location of the arch central point, or the interincisal point, a line of diameter extending beyond the circle was drawn. The length of the said line corresponded to the arch width between the molars. A distance corresponding to the dental arch depth, was taken on the constructed diameter. Through the depth's mark a perpendicular line was drawn, and on both sides of the center distances were measured corresponding to arch half-width between the molars. From the interincisal point, on both sides of the arch, a value equal to the anterior section diagonal was used to measure segments thus obtaining a reference point for the canines positions. Through the lower point, which was on the diameter line, a perpendicular line was drawn with two segments measured, equal to the one-and-a-half width of the tooth-alveolar arch in the second primary molars area. The obtained points were used as reference for constructing arch segments from the canine point to the molar point. This way we obtained an arch diagram corresponding to the individual parameters of the primary occlusion dental arches.

Conclusion. The biometric examination of dental arches on jaw stone models allowed develop- 
ing a mathematical simulation method to build an upper dental arch diagram for primary occlusion. The technique is based on the regularities of the circle geometry, where the diameter is defined as the ratio of the arch width between the canines and the central angle sine. The value of the central angle here, according to the circle geometry laws, was the ratio of the arch depth double arctangent to the width between the primary canines. Overlapping the diagram on the upper dental arch model showed their match, which allowed applying the method of arch constructing in clinical orthodontic practice.

\section{REFERENCES}

1. DMitrienko S.V., Domenyuk D.A., Vedeshina E.G. Shape individualization in lower dental arches drawn on basic morphometric features // Archiv EuroMedica, 2015. - Vol. 5. - № 1. - P. 11-15.

2. Domenyuk D.A., Vedeshina E.G., Dmitrienko S.V. Dynamics of changes in transverse and frontal- retromolar jaw sizes in children throughout the orthodontic treatment stages. Kubanskij nauchnyj medicinskij vestnik. 2016;(3):51-59. (In Russ.) DOI:10.25207/1608-6228-2016-3-51-59.

3. Domenyuk D.A., Shkarin V.V., Porfiriadis M.P., DMitrienko D.S., Dmitrienko S.V. Classification of facial types in view of gnathology // Archiv EuroMedica, 2017. - Vol. 7. - № 1. - P. 8-13.

4. DomenYuk, D.A. Modern classification of dental arches / D.A. Domenyuk, S.V. Dmitrienko // Archiv EuroMedica, 2014. - Vol. 4. - № 2. - P. 14-16.

5. Shkarin V.V., Domenyuk D.A., Porfiriadis M.P., DMitrienko D.S., DMitrienko S.V. Mathematical and graphics simulation for individual shape of maxillary dental arch // Archiv EuroMedica, 2017. - Vol. 7. - № 1. - C. 60-65.

6. ShKarin V.V., Porfiriadis M.P., Domenyuk D.A., DMitrienko D.S., DMitrienko S.V. Setting reference points for key teeth location in case of abnormal dental arch shape //Archiv EuroMedica. - 2017. - Vol. 7. - № 2. - P. 111-117.

\section{DIAGNOSTIC VALUE OF CEPHALOMETRIC PARAMETERS AT GRAPHIC REPRODUCTION OF TOOTH DENTAL ARCHES IN PRIMARY TEETH OCCLUSION}

\section{Alexander Lepilin, Igor Fomin, Dmitry Domenyuk, Sergei Dmitrienko, Gasan Budaychiev}

One of the significant and objective ways for diagnosing anomalies in the dentoalveolar system, as well as for controlling the treatment effectiveness and forecasting potential aesthetic changes in the face soft tissue base, implies studying the cephalometric parameters. Diagnosis is given based on clinical and radiological examination, as well as on studying control and diagnos-tic jaw models $[1,3]$. There are findings available, which show odontometry of infant teeth and linear parameters of dental arches, as well as methods have been proposed for graphic reproduc-tion of dental arches in view of individual features $[2,5]$.

The Schwarz method for designing a semicircle has been proposed, where the diameter is constituted by the width of the dental arch between the vestibular surfaces of the second primary molars. The specific features for constructing the dental arch of the primary occlusion have been shown, taking into account the lateral segment length, which includes the sizes of the primary molars and the canine. Notable are works claiming potential employment of the circle geometry regularities for constructing individual shape of dental arches in the permanent teeth occlusion, in view of their gnathic and dental types $[4,6]$. However, there is no data available on methods for diagnosing dentoalveolar anomalies in the primary teeth occlusion, taking into account the parameters of the facial part of the head.

Arm. Identifying the face and the dental arches basic parameters in children at their primary occlusion stage in order to develop methods for diagnosing maxillofacial anomalies.

Materials and methods. Anthropometric examination of the face and dental arches was carried out involving 67 children (aged 4-6) with a full set of primary teeth without signs of maxillofacial pathology. The anthropometric examination of the face was performed in the vertical direction from the superciliary point (g-glabella) to the nasal point of the subnasale (sn), and from that to the chin point gnathion (gn). This way the vertical dimensions were obtained for the middle (nasal) and lower (gnathic) part of the face. The transversal direction was used to 